\title{
CONSTRAINT-PRESERVING ENERGY-STABLE SCHEME FOR THE 2D SIMPLIFIED ERICKSEN-LESLIE SYSTEM*
}

\author{
Xuelian Bao \\ School of Mathematical Sciences, Beijing Normal University, Beijing 100875, China; \\ Email: xuelian_bao@163.com \\ Rui Chen \\ School of Science, Beijing University of Posts and Telecommunications, Beijing 100876, China; \\ Email: ruichen@bupt.edu.cn \\ Hui Zhang \\ Laboratory of Mathematics and Complex Systems, Ministry of Education \\ and School of Mathematical Sciences, Beijing Normal University, Beijing 100875, China; \\ Email: hzhang@bnu.edu.cn
}

\begin{abstract}
Here we consider the numerical approximations of the 2D simplified Ericksen-Leslie system. We first rewrite the system and get a new system. For the new system, we propose an easy-to-implement time discretization scheme which preserves the sphere constraint at each node, enjoys a discrete energy law, and leads to linear and decoupled elliptic equations to be solved at each time step. A discrete maximum principle of the scheme in the finite element form is also proved. Some numerical simulations are performed to validate the scheme and simulate the dynamic motion of liquid crystals.
\end{abstract}

Mathematics subject classification: 52B10, 65D18, 68U05, 68U07.

Key words: Nematic liquid crystal, Ericksen-Leslie system, Constraint preserving, Finite element.

\section{Introduction}

Here we consider the 2D simplified Ericksen-Leslie system which models the hydrodynamics of nematic liquid crystals. The system is a simplified version of the Ericksen-Leslie system introduced by Ericksen [12] and Leslie [20]. Since the full Ericksen-Leslie system is too complicated, Lin [21] proposed this simplified version in 1989. The model is derived as the following coupled system:

$$
\begin{aligned}
& \mathbf{d}_{t}+(\mathbf{u} \cdot \nabla) \mathbf{d}=\Delta \mathbf{d}+|\nabla \mathbf{d}|^{2} \mathbf{d}, \\
& |\mathbf{d}|=1 \\
& \mathbf{u}_{t}+\mathbf{u} \cdot \nabla \mathbf{u}+\nabla P=\Delta \mathbf{u}-\nabla \cdot\left((\nabla \mathbf{d})^{T} \nabla \mathbf{d}\right), \\
& \nabla \cdot \mathbf{u}=0
\end{aligned}
$$

Here, $\Omega$ is a bounded domain in $\mathbb{R}^{2}$, the given time $T>0 . \mathbf{u}, \mathbf{d}: \Omega \times[0, T] \rightarrow \mathbb{R}^{2}$ are the fluid velocity and the mean orientation of the molecules respectively, $P: \Omega \times[0, T] \rightarrow \mathbb{R}$ is the fluid pressure. Equation (1.3) is the Navier-Stokes equation [31] coupled with the extra term

\footnotetext{
* Received July 10, 2018 / Revised version received May 14, 2019 / Accepted June 25, 2019 /
}

Published online January 6, 2020 / 
$\nabla \cdot\left((\nabla \mathbf{d})^{T} \nabla \mathbf{d}\right)$, and equation (1.1) is the harmonic map heat flow with the convection term $(\mathbf{u} \cdot \nabla) \mathbf{d}[25]$.

We will investigate the system with homogeneous Dirichlet boundary conditions for the velocity field and homogeneous Neumann boundary conditions for the director field:

$$
\mathbf{u}=0, \quad \frac{\partial \mathbf{d}}{\partial \mathbf{n}}=\mathbf{0}, \quad \text { on } \quad \partial \Omega \times(0, T),
$$

where $\mathbf{n}$ denotes the outer normal vector on the boundary.

The initial conditions are used as follows:

$$
\mathbf{d}(\mathbf{x}, 0)=\mathbf{d}_{0}(\mathbf{x}), \quad \mathbf{u}(\mathbf{x}, 0)=\mathbf{u}_{0}(\mathbf{x}), \quad \text { in } \quad \Omega,
$$

where $\mathbf{u}_{0}: \Omega \rightarrow \mathbb{R}^{2}$ satisfying $\nabla \cdot \mathbf{u}_{0}=0$, and $\mathbf{d}_{0}: \Omega \rightarrow \mathbb{R}^{2}$ satisfying $\left|\mathbf{d}_{0}\right|=1$ are given functions. Under the boundary conditions mentioned above, the system (1.1)-(1.4) satisfies the following energy law:

$$
\frac{d}{d t}\left(\frac{1}{2}\|\mathbf{u}\|^{2}+\frac{1}{2}\|\nabla \mathbf{d}\|^{2}\right)+\|\nabla \mathbf{u}\|^{2}+\left\|\Delta \mathbf{d}+|\nabla \mathbf{d}|^{2} \mathbf{d}\right\|^{2}=0,
$$

where $\|\cdot\|$ denotes the $L^{2}$ norm in $\Omega$.

It requires that $\mathbf{d}$ must have the unit length, i.e., $|\mathbf{d}|=1$ almost everywhere. From the numerical point of view, this constraint makes it difficult to manage since we can not imply the sphere constraint at the nodes via interpolation. In addition, the presence of the extra term $\nabla \cdot\left((\nabla \mathbf{d})^{T} \nabla \mathbf{d}\right)$ causes strong coupling [27]. Hence, a penalty function such as the GinzburgLandau approximation is widely used to overcome these difficulties [22], and the general penalty version reads as follows:

$$
\begin{aligned}
& \mathbf{d}_{t}+(\mathbf{u} \cdot \nabla) \mathbf{d}+\frac{1}{\epsilon^{2}} \mathbf{f}(\mathbf{d})-\Delta \mathbf{d}=\mathbf{0}, \\
& \mathbf{u}_{t}+\mathbf{u} \cdot \nabla \mathbf{u}+\nabla P=\Delta \mathbf{u}-\nabla \cdot\left((\nabla \mathbf{d})^{T} \nabla \mathbf{d}\right), \\
& \nabla \cdot \mathbf{u}=0
\end{aligned}
$$

where $\epsilon>0$ is the penalty parameter, $\mathbf{f}(\mathbf{d})$ is the Ginzburg-Landau approximation of the constraint $|\mathbf{d}|=1$ for small $\epsilon$. The penalty function is the gradient of a scalar-valued function $F(\mathbf{d})$, i.e., $\mathbf{f}(\mathbf{d})=\nabla_{\mathbf{d}} F(\mathbf{d})$, where,

$$
F(\mathbf{d})= \begin{cases}\frac{1}{4}\left(|\mathbf{d}|^{2}-1\right)^{2}, & \text { if }|\mathbf{d}| \leqslant 1 \\ \left(|\mathbf{d}|^{2}-1\right)^{2}, & \text { if }|\mathbf{d}|>1\end{cases}
$$

It is still an open problem that whether weak solutions $\left(\mathbf{u}_{\epsilon}, \mathbf{d}_{\epsilon}\right)$ of the system (1.8)-(1.10) with Dirichlet boundary conditions weakly converge to that of the system (1.1)-(1.4) as $\epsilon \rightarrow 0$ [27]. It has been proved that, up to a subsequence, $\left(\mathbf{u}_{\epsilon}, \mathbf{d}_{\epsilon}\right)$ weakly converge to $(\mathbf{u}, \mathbf{d})$ which satisfies a system the same as (1.1)-(1.4) except for an additional measure-valued tensor in the equation (1.3) [24].

In [22], Lin and Liu proved the global existence of the solution of (1.8)-(1.10) with Dirichlet boundary conditions in the dimension two and three. Later, Lin and Liu in [23] proved partial regularity of weak solutions to the system in the dimension three. Since the Ericksen-Leslie system with $|\mathbf{d}|=1$ is complicated, it was a challenging problem to prove global existence of 
solutions to the system (1.1)-(1.4). In the 2D case, the existence was obtained by Lin-Lin-Wang [25], where the domain should be bounded and smooth. The associated uniqueness problem was later studied in [26]. In [17], Hong considered the same system but on the whole space $\mathbb{R}^{2}$. Lin-Lin-Wang and Hong also showed the partial regularity of the global weak solutions, which are smooth away from possibly finitely many points. In the 3D case, by giving a representation formula for the solutions of incompressible liquid crystal flow in arbitrary dimensions, Huang [19] obtained a unique strong global solution to the system (1.1)-(1.4).

Recently, many mathematicians are absorbed in investigating solutions of the EricksenLeslie system. Based on the finite time singularities of the 2D heat flow of harmonic maps [6], solutions of (1.1)-(1.4) with finite time singularities have been recently constructed in [18], where the spatial domain is a bounded open set in $\mathbb{R}^{3}$. The long time behavior of solutions of (1.1)-(1.4) is concerned in $[9,10]$. The behavior of defects is also an important subject in the study of liquid crystals. The Ericksen-Leslie theory can be used to study the motion of point defects. Readers can refer to [8] and references therein for more details. Some numerical simulations of point defects in the Ericksen-Leslie theory can be found in $[3,5,8,28,29]$.

The sphere constraint $|\mathbf{d}|=1$ is difficult to achieve at the discrete level, therefore many numerical studies for the system (1.1)-(1.4) are based on the discretization of the penalized problem (1.8)-(1.10) (see e.g., [3, $5,14,28,29])$. Readers can refer to [1] and references therein for more details. In order to achieve the sphere constraint, based on a convergent discretization of the harmonic map heat flow without the convection term [2] (i.e., (1.1) with $\mathbf{u}=\mathbf{0}$ ), Becker proposed an energy-stable and constraint-preserving scheme directly discretizing (1.1)-(1.4) in [3]. However, solvability of the scheme requires restricted mesh parameters. And when the discrete time and space parameters go to zero, the convergence is an open problem. In [13], a nonlinear constraint-preserving finite difference scheme for the 2D system (1.1)-(1.4) is proposed, and the unique solvability is also gained.

The goal of this paper is to design a stabilized, decoupled, constraint-preserving scheme for the system (1.1)-(1.4) in the dimension two. Inspired by $[11,15,19]$, we rewrite the system (1.1)-(1.4) in the dimension two by denoting $\mathbf{d}(\mathbf{x}, t)=\left(d_{1}, d_{2}\right)^{T}$ as

$$
\mathbf{d}(\mathbf{x}, t)=(\cos \theta(\mathbf{x}, t), \sin \theta(\mathbf{x}, t))^{T},
$$

where $\theta(\mathbf{x}, t)=\arg \mathbf{d}(\mathbf{x}, t)$ with arg being the argument from the polar coordinates, and get a new system of functions $\theta, \mathbf{u}$ and $P$. Inspired by [7], we design a numerical scheme for the new system. The main features of our scheme include the following: (i) it satisfies the sphere constraint for $\mathbf{d}$ at each node; (ii) it is unconditionally solvable and satisfies a discrete energy law; (iii) it leads to decoupled elliptic equations to solve at each time step which is easy to implement; (iv) it satisfies a discrete maximum principle in the finite element form under some conditions so that equivalence between the new system and the system (1.1)-(1.4) is ensured when we perform numerical experiments.

This paper is organized as follows. In Section 2, we rewrite the 2D simplified EricksenLeslie system and derive the energy law. In Section 3, we construct a decoupled, energy stable numerical scheme for the new system, which satisfies a discrete energy law. In Section 4, we present the spatial discretization using the finite element method. In Section 5 , we prove the discrete maximum principle of the scheme in the finite element form. In Section 6, we present some computational experiments to validate the scheme and simulate the dynamic motion of liquid crystals. Some concluding remarks are given in Section 7 . 


\section{Alternative Formulation and Energy Law}

In this section, we first show that the sphere constraint $|\mathbf{d}|=1$ is satisfied automatically due to the equation (1.1) if $\mathbf{d}$ is smooth. Then we rewrite the system (1.1)-(1.4), and establish the new energy law.

Theorem 2.1. Assume that $(\mathbf{u}, \mathbf{d})$ is the smooth solution of the following equation:

$$
\mathbf{d}_{t}+(\mathbf{u} \cdot \nabla) \mathbf{d}=\Delta \mathbf{d}+|\nabla \mathbf{d}|^{2} \mathbf{d},
$$

in $\Omega \times(0, T)$ with $\mathbf{d}(\mathbf{x}, 0)=\mathbf{d}_{0}(\mathbf{x})$. If the initial condition satisfies $\left|\mathbf{d}_{0}\right|=1$, then $|\mathbf{d}|=1$ for all $t$ belonging to $(0, T)$.

Proof. Multiplying both sides of (2.1) by $\mathbf{d}$, we obtain

$$
\frac{1}{2} \frac{\partial}{\partial t}|\mathbf{d}|^{2}+\mathbf{u} \cdot \nabla \frac{|\mathbf{d}|^{2}}{2}=\Delta \frac{|\mathbf{d}|^{2}}{2}-|\nabla \mathbf{d}|^{2}+|\nabla \mathbf{d}|^{2}|\mathbf{d}|^{2} .
$$

Thus, changing the formula yields

$$
\frac{\partial}{\partial t} \frac{|\mathbf{d}|^{2}-1}{2}+\mathbf{u} \cdot \nabla \frac{|\mathbf{d}|^{2}-1}{2}=\triangle \frac{|\mathbf{d}|^{2}-1}{2}+|\nabla \mathbf{d}|^{2}\left(|\mathbf{d}|^{2}-1\right) .
$$

Denoting $v=\frac{|\mathbf{d}|^{2}-1}{2}$, we have

$$
\frac{\partial}{\partial t} v+\mathbf{u} \cdot \nabla v=\Delta v+2|\nabla \mathbf{d}|^{2} v
$$

According to the maximum principle [30], $|v| \leqslant\left|v_{0}\right|$, i.e., $\left.|| \mathbf{d}\right|^{2}-1|\leqslant|\left|\mathbf{d}_{0}\right|-1 \mid$.

Since $\left|\mathbf{d}_{0}\right|=1$, finally, we obtain $|\mathbf{d}|=1$.

Hence, we can write the orientation field vector $\mathbf{d}$ in the polar coordinate to rewrite the system (1.1)-(1.4), i.e.,

$$
\mathbf{d}(\mathbf{x}, t)=(\cos \theta(\mathbf{x}, t), \sin \theta(\mathbf{x}, t))^{T},
$$

where $\mathbf{x}=(x, y) \in \Omega \subset \mathbb{R}^{2}$. Here, $\theta(\mathbf{x}, t)=\arg \mathbf{d}(\mathbf{x}, t)$, arg is the argument from the polar coordinates and $\theta(\mathbf{x}, t) \in[0,2 \pi)$. Then

$$
\begin{aligned}
& \mathbf{d}_{t}=\left(d_{1 \theta} \theta_{t}, d_{2 \theta} \theta_{t}\right)^{T}=(-\sin \theta, \cos \theta)^{T} \theta_{t}, \\
& \nabla \mathbf{d}=\left[\begin{array}{ll}
d_{1 \theta} \theta_{x} & d_{1 \theta} \theta_{y} \\
d_{2 \theta} \theta_{x} & d_{2 \theta} \theta_{y}
\end{array}\right]=\left[\begin{array}{cc}
-\sin \theta \theta_{x} & -\sin \theta \theta_{y} \\
\cos \theta \theta_{x} & \cos \theta \theta_{y}
\end{array}\right]=\left(\begin{array}{c}
-\sin \theta \\
\cos \theta
\end{array}\right)\left(\theta_{x}, \theta_{y}\right), \\
& |\nabla \mathbf{d}|^{2}=\theta_{x}^{2}+\theta_{y}^{2} \\
& \Delta d_{1}=\left(d_{1 \theta} \theta_{x}\right)_{x}+\left(d_{1 \theta} \theta_{y}\right)_{y}=-\cos \theta|\nabla \theta|^{2}-\sin \theta \Delta \theta \\
& \Delta d_{2}=\left(d_{2 \theta} \theta_{x}\right)_{x}+\left(d_{2 \theta} \theta_{y}\right)_{y}=-\sin \theta|\nabla \theta|^{2}+\cos \theta \Delta \theta, \\
& \Delta \mathbf{d}=\left(\Delta d_{1}, \Delta d_{2}\right)^{T}=\Delta \theta(-\sin \theta, \cos \theta)^{T}-\left(\theta_{x}^{2}+\theta_{y}^{2}\right)(\cos \theta, \sin \theta)^{T}
\end{aligned}
$$

Hence,

$$
\begin{aligned}
& \Delta \mathbf{d}+|\nabla \mathbf{d}|^{2} \mathbf{d} \\
= & \Delta \theta(-\sin \theta, \cos \theta)^{T}-\left(\theta_{x}^{2}+\theta_{y}^{2}\right)(\cos \theta, \sin \theta)^{T}+\left(\theta_{x}^{2}+\theta_{y}^{2}\right)(\cos \theta, \sin \theta)^{T} \\
= & \Delta \theta(-\sin \theta, \cos \theta)^{T} .
\end{aligned}
$$


Moreover, observing that

$$
(\nabla \mathbf{d})^{T} \Delta \mathbf{d}=\left(\theta_{x}, \theta_{y}\right)^{T} \Delta \theta
$$

we have

$$
\nabla \cdot\left((\nabla \mathbf{d})^{T} \nabla \mathbf{d}\right)=(\nabla \mathbf{d})^{T} \Delta \mathbf{d}+\frac{1}{2} \nabla\left(|\nabla \mathbf{d}|^{2}\right)=\left(\theta_{x}, \theta_{y}\right)^{T} \Delta \theta+\frac{1}{2} \nabla\left(|\nabla \theta|^{2}\right) .
$$

Eq. (1.1) can be rewritten as:

$$
(-\sin \theta, \cos \theta) \theta_{t}+\mathbf{u} \cdot \nabla \theta(-\sin \theta, \cos \theta)=\Delta \theta(-\sin \theta, \cos \theta) .
$$

The whole system (1.1)-(1.4), denoting $\tilde{P}=P+\frac{1}{2}|\nabla \theta|^{2}$ and $\theta \in[0,2 \pi)$, reads as:

$$
\begin{aligned}
& \theta_{t}+(\mathbf{u} \cdot \nabla) \theta-\Delta \theta=0 \\
& \mathbf{u}_{t}+\mathbf{u} \cdot \nabla \mathbf{u}+\nabla \tilde{P}-\Delta \mathbf{u}+\Delta \theta \nabla \theta=0 \\
& \nabla \cdot \mathbf{u}=0
\end{aligned}
$$

To these equations we add the following boundary and initial conditions which are derived from (1.5) and (1.6) respectively:

$$
\begin{aligned}
& \mathbf{u}=0, \quad \nabla \theta \cdot \mathbf{n}=0, \quad \text { on } \quad \partial \Omega \times(0, T), \\
& \theta(\mathbf{x}, 0)=\theta_{0}(\mathbf{x}), \quad \mathbf{u}(\mathbf{x}, 0)=\mathbf{u}_{0}(\mathbf{x}), \quad \text { in } \Omega,
\end{aligned}
$$

where $\mathbf{u}_{0}: \Omega \rightarrow \mathbb{R}^{2}$ satisfying $\nabla \cdot \mathbf{u}_{0}=0$, and $\theta_{0}: \Omega \rightarrow[0,2 \pi)$ are given functions.

The next theorem derives the energy law associated with the system (2.6)-(2.8).

Theorem 2.2. The system (2.6)-(2.8) satisfies the following energy law:

$$
\frac{d}{d t} E+\|\Delta \theta\|^{2}+\|\nabla \mathbf{u}\|^{2}=0
$$

where $E=\frac{1}{2}\|\mathbf{u}\|^{2}+\frac{1}{2}\|\nabla \theta\|^{2}$.

Proof. We can rewrite (2.7) by using (2.6):

$$
\mathbf{u}_{t}+\mathbf{u} \cdot \nabla \mathbf{u}+\nabla \tilde{P}-\Delta \mathbf{u}+\left(\theta_{t}+(\mathbf{u} \cdot \nabla) \theta\right) \nabla \theta=0 .
$$

Multiplying $(2.6)$ by $\left(-\theta_{t}\right)$ and integrating over $\Omega$, we get

$$
\frac{d}{d t}\left(\frac{1}{2}\|\nabla \theta\|^{2}\right)=-\left\|\theta_{t}+(\mathbf{u} \cdot \nabla) \theta\right\|^{2}+\left((\mathbf{u} \cdot \nabla) \theta, \theta_{t}+(\mathbf{u} \cdot \nabla) \theta\right),
$$

where $(\cdot, \cdot)$ denotes the inner product in $\Omega$. Multiplying $(2.12)$ by $\mathbf{u}$ and integrating over $\Omega$, we get

$$
\frac{d}{d t}\left(\frac{1}{2}\|\mathbf{u}\|^{2}\right)+\|\nabla \mathbf{u}\|^{2}+\left(\left(\theta_{t}+(\mathbf{u} \cdot \nabla) \theta\right) \nabla \theta, \mathbf{u}\right)=0
$$

Combining these two equations above, we can get the energy law:

$$
\frac{d}{d t}\left(\frac{1}{2}\|\mathbf{u}\|^{2}+\frac{1}{2}\|\nabla \theta\|^{2}\right)+\left\|\theta_{t}+(\mathbf{u} \cdot \nabla) \theta\right\|^{2}+\|\nabla \mathbf{u}\|^{2}=0 .
$$

This completes the proof of the theorem. 
Remark 2.1. The new system does not need an extra constraint $|\mathbf{d}|=1$ and it is computationally cheaper. One only needs to calculate the scalar function $\theta(\mathbf{x}, t)$ instead of the vector function $\mathbf{d}(\mathbf{x}, t)$ which has two components.

Remark 2.2. Due to the definition of function $\theta(\mathbf{x}, t)$, it is obvious that $\theta(\mathbf{x}, t) \in[0,2 \pi)$ and there is a one-to-one correspondence between function $\mathbf{d}(\mathbf{x}, t)$ and function $\theta(\mathbf{x}, t)$, so that system (2.6)-(2.8) is equivalent to system (1.1)-(1.4).

If we assume that $\mathbf{u}$ is bounded, according to the maximum principle [30] for equation (2.6), if the initial data $\theta_{0} \in[0,2 \pi)$, then function $\theta(\mathbf{x}, t) \in[0,2 \pi)$. Hence, in order to ensure $\theta(\mathbf{x}, t) \in[0,2 \pi)$ and obtain the equivalence between the two systems, we only need to let $\theta_{0} \in[0,2 \pi)$.

Remark 2.3. The penalized system (1.8)-(1.10) can describe the cases where the orientation field $\mathbf{d}$ has singular points, which are defined as the points where $|\mathbf{d}|=0$ [3]. However, the system (2.6)-(2.8) is not suitable to describe these singular points since $|\mathbf{d}| \equiv 1$.

\section{Energy Stable Scheme}

This section is devoted to constructing a decoupled energy-stable scheme for the system (2.6)-(2.8) and proving the discrete energy law. Our numerical scheme reads as follows.

\section{Algorithm 3.1.}

Given the initial conditions $\theta^{0}, \mathbf{u}^{0}$ and $P^{0}$, having computed for $\theta^{n}, \mathbf{u}^{n}, P^{n}$ and $\tilde{P}^{n}=P^{n}+\frac{1}{2}\left|\nabla \theta^{n}\right|^{2}$ for $n>0$, we compute $\theta^{n+1}, \mathbf{u}^{n+1}, P^{n+1}$ by

Step 1.

$$
\frac{\theta^{n+1}-\theta^{n}}{\delta t}+\left(\mathbf{u}_{*}^{n} \cdot \nabla\right) \theta^{n}=\Delta \theta^{n+1},
$$

where

$$
\mathbf{u}_{*}^{n}=\mathbf{u}^{n}-\delta t\left[\frac{\theta^{n+1}-\theta^{n}}{\delta t}+\left(\mathbf{u}_{*}^{n} \cdot \nabla\right) \theta^{n}\right] \nabla \theta^{n} .
$$

Step 2.

$$
\begin{aligned}
& \frac{\tilde{\mathbf{u}}^{n+1}-\mathbf{u}_{*}^{n}}{\delta t}+\left(\mathbf{u}^{n} \cdot \nabla\right) \tilde{\mathbf{u}}^{n+1}-\Delta \tilde{\mathbf{u}}^{n+1}+\nabla \tilde{P}^{n}=0, \\
& \left.\tilde{\mathbf{u}}^{n+1}\right|_{\partial \Omega}=0 .
\end{aligned}
$$

Step 3.

$$
\begin{aligned}
& \frac{\mathbf{u}^{n+1}-\tilde{\mathbf{u}}^{n+1}}{\delta t}+\nabla\left(\tilde{P}^{n+1}-\tilde{P}^{n}\right)=0, \\
& \nabla \cdot \mathbf{u}^{n+1}=0 \\
& \left.\mathbf{u}^{n+1} \cdot \mathbf{n}\right|_{\partial \Omega}=0 .
\end{aligned}
$$

And

$$
P^{n+1}=\tilde{P}^{n+1}-\frac{1}{2}\left|\nabla \theta^{n+1}\right|^{2} .
$$


Remark 3.1. We use the pressure-correction scheme [16] to decouple the computation of the pressure from that of the velocity.

Remark 3.2. We introduce $\mathbf{u}_{*}^{n}$ to decouple the equation (2.6). It can be computed directly from (3.2), i.e.,

$$
\mathbf{u}_{*}^{n}=\left(I+\delta t \nabla \theta^{n}\left(\nabla \theta^{n}\right)^{T}\right)^{-1}\left[\mathbf{u}^{n}-\left(\theta^{n+1}-\theta^{n}\right) \nabla \theta^{n}\right]
$$

It is easy to see that the matrix $I+\delta t \nabla \theta^{n}\left(\nabla \theta^{n}\right)^{T}$ is invertible. A similar term was used in [7] for a phase-field vesicle membrane model.

Remark 3.3. The scheme (3.1)-(3.7) is a totally decoupled linear scheme and is first order in time. (3.1) can be transformed into a second order elliptic equation which is shown in Section 4. Step 3 can be transformed into a Poisson equation for $\tilde{P}^{n+1}-\tilde{P}^{n}$. Hence, at each time step, we only need to solve three decoupled elliptic equations.

Remark 3.4. We can prove that the above scheme is unconditionally energy stable, see (3.10).

Here we establish the discrete energy law which exactly mimics the differential energy law $(2.11)$.

Theorem 3.1. The scheme (3.1)-(3.7) is stable, with the following discrete energy dissipation law:

$$
\begin{aligned}
& E^{n+1}+\frac{\delta t^{2}}{2}\left\|\nabla \tilde{P}^{n+1}\right\|^{2}+\delta t\left\|\frac{\theta^{n+1}-\theta^{n}}{\delta t}+\left(\mathbf{u}_{*} \cdot \nabla\right) \theta^{n}\right\|^{2}+\delta t\left\|\nabla \tilde{\mathbf{u}}^{n+1}\right\|^{2} \\
\leq & E^{n}+\frac{\delta t^{2}}{2}\left\|\nabla \tilde{P}^{n}\right\|^{2}
\end{aligned}
$$

where $E^{n}=\frac{1}{2}\left\|\mathbf{u}^{n}\right\|^{2}+\frac{1}{2}\left\|\nabla \theta^{n}\right\|^{2}$.

Proof. By taking the inner product of (3.1) with $\left(-\frac{\theta^{n+1}-\theta^{n}}{\delta t}\right)$, and using the identity

$$
2(a-b, a)=|a|^{2}-|b|^{2}+|a-b|^{2},
$$

we obtain

$$
\begin{aligned}
& \frac{1}{2 \delta t}\left(\left\|\nabla \theta^{n+1}\right\|^{2}-\left\|\nabla \theta^{n}\right\|^{2}+\left\|\nabla \theta^{n+1}-\nabla \theta^{n}\right\|^{2}\right) \\
= & -\left\|\frac{\theta^{n+1}-\theta^{n}}{\delta t}+\left(\mathbf{u}_{*}^{n} \cdot \nabla\right) \theta^{n}\right\|^{2}+\left(\mathbf{u}_{*}^{n} \cdot \nabla \theta^{n}, \frac{\theta^{n+1}-\theta^{n}}{\delta t}+\left(\mathbf{u}_{*}^{n} \cdot \nabla\right) \theta^{n}\right) .
\end{aligned}
$$

We derive from (3.2) that

$$
\frac{\mathbf{u}_{*}^{n}-\mathbf{u}^{n}}{\delta t}=-\left(\frac{\theta^{n+1}-\theta^{n}}{\delta t}+\mathbf{u}_{*}^{n} \cdot \nabla \theta^{n}\right) \nabla \theta^{n} .
$$

Then by taking the inner product of $(3.12)$ with $\mathbf{u}_{*}^{n}$, we obtain

$$
\frac{1}{2 \delta t}\left(\left\|\mathbf{u}_{*}^{n}\right\|^{2}-\left\|\mathbf{u}^{n}\right\|^{2}+\left\|\mathbf{u}_{*}^{n}-\mathbf{u}^{n}\right\|^{2}\right)=-\left(\left(\frac{\theta^{n+1}-\theta^{n}}{\delta t}+\mathbf{u}_{*}^{n} \cdot \nabla \theta^{n}\right) \nabla \theta^{n}, \mathbf{u}_{*}^{n}\right) .
$$

By the same way as (3.3) with $\tilde{\mathbf{u}}^{n+1}$, we obtain

$$
\frac{1}{2 \delta t}\left(\left\|\tilde{\mathbf{u}}^{n+1}\right\|^{2}-\left\|\mathbf{u}_{*}^{n}\right\|^{2}+\left\|\tilde{\mathbf{u}}^{n+1}-\mathbf{u}_{*}^{n}\right\|^{2}\right)+\left\|\nabla \tilde{\mathbf{u}}^{n+1}\right\|^{2}+\left(\nabla \tilde{P}^{n}, \tilde{\mathbf{u}}^{n+1}\right)=0 .
$$


Similarly, taking the inner product of (3.5) with $\mathbf{u}^{n+1}$, we obtain

$$
\frac{1}{2 \delta t}\left(\left\|\mathbf{u}^{n+1}\right\|^{2}-\left\|\tilde{\mathbf{u}}^{n+1}\right\|^{2}+\left\|\mathbf{u}^{n+1}-\tilde{\mathbf{u}}^{n+1}\right\|^{2}\right)=0 .
$$

To deal with the pressure term, we take the inner product of (3.5) with $\delta t \nabla \tilde{P}^{n}$ to derive

$$
\frac{1}{2} \delta t\left(\left\|\nabla \tilde{P}^{n+1}\right\|^{2}-\left\|\nabla \tilde{P}^{n}\right\|^{2}-\left\|\nabla \tilde{P}^{n+1}-\nabla \tilde{P}^{n}\right\|^{2}\right)=\left(\nabla \tilde{P}^{n}, \tilde{\mathbf{u}}^{n+1}\right) .
$$

We also derive from (3.5) directly that

$$
\frac{1}{2} \delta t\left\|\nabla \tilde{P}^{n+1}-\nabla \tilde{P}^{n}\right\|^{2}=\frac{1}{2 \delta t}\left\|\mathbf{u}^{n+1}-\tilde{\mathbf{u}}^{n+1}\right\|^{2} .
$$

Combining all the identities above, we obtain

$$
\begin{aligned}
& \frac{1}{2 \delta t}\left(\left\|\nabla \theta^{n+1}\right\|^{2}+\left\|\mathbf{u}^{n+1}\right\|^{2}\right)+\frac{1}{2} \delta t\left\|\nabla \tilde{P}^{n+1}\right\|^{2}-\frac{1}{2 \delta t}\left(\left\|\nabla \theta^{n}\right\|^{2}+\left\|\mathbf{u}^{n}\right\|^{2}\right)-\frac{1}{2} \delta t\left\|\nabla \tilde{P}^{n}\right\|^{2} \\
= & -\frac{1}{2 \delta t}\left(\left\|\nabla \theta^{n+1}-\nabla \theta^{n}\right\|^{2}+\left\|\tilde{\mathbf{u}}^{n+1}-\mathbf{u}_{*}^{n}\right\|^{2}+\left\|\mathbf{u}_{*}^{n}-\mathbf{u}^{n}\right\|^{2}\right) \\
& -\left\|\frac{\theta^{n+1}-\theta^{n}}{\delta t}+\mathbf{u}_{*}^{n} \cdot \nabla \theta^{n}\right\|^{2}-\left\|\nabla \tilde{\mathbf{u}}^{n+1}\right\|^{2}
\end{aligned}
$$

Then we can get the energy law (3.10).

\section{Spatial Discretization}

We use the finite element method for the spatial discretization here.

\subsection{Weak forms}

We rewrite the equation (3.2) as follows:

$$
\mathbf{u}_{*}^{n}=\left(A^{n}\right)^{-1}\left[\mathbf{u}^{n}-\left(\theta^{n+1}-\theta^{n}\right) \nabla \theta^{n}\right]
$$

with

$$
A^{n}=I+\delta t \nabla \theta^{n}\left(\nabla \theta^{n}\right)^{T},
$$

where $I$ is the unit matrix. So the equation can be transformed into:

$$
\begin{aligned}
& \Delta \theta^{n+1}+\left\{\left[\left(A^{n}\right)^{-1} \nabla \theta^{n}\right] \cdot \nabla \theta^{n}-\frac{1}{\delta t}\right\} \theta^{n+1} \\
= & \theta^{n}\left[\left(A^{n}\right)^{-1} \nabla \theta^{n}\right] \cdot \nabla \theta^{n}+\left[\left(A^{n}\right)^{-1} \mathbf{u}^{n}\right] \cdot \nabla \theta^{n}-\frac{1}{\delta t} \theta^{n} .
\end{aligned}
$$

Denote

$$
\begin{aligned}
& a^{n}=\left[\left(A^{n}\right)^{-1} \nabla \theta^{n}\right] \cdot \nabla \theta^{n}-\frac{1}{\delta t}, \\
& b^{n}=\theta^{n}\left[\left(A^{n}\right)^{-1} \nabla \theta^{n}\right] \cdot \nabla \theta^{n}+\left[\left(A^{n}\right)^{-1} \mathbf{u}^{n}\right] \cdot \nabla \theta^{n}-\frac{1}{\delta t} \theta^{n} .
\end{aligned}
$$

By simple calculation, we can get $\left|A^{n}\right|=\delta t\left|\nabla \theta^{n}\right|^{2}+1$,

$$
\left(A^{n}\right)^{-1}=\frac{1}{\delta t\left|\nabla \theta^{n}\right|^{2}+1}\left[I+\delta t\left(\theta_{y}^{n},-\theta_{x}^{n}\right)^{T}\left(\theta_{y}^{n},-\theta_{x}^{n}\right)\right] .
$$


Then we have

$$
\begin{aligned}
a^{n} & =\left[\left(A^{n}\right)^{-1} \nabla \theta^{n}\right] \cdot \nabla \theta^{n}-\frac{1}{\delta t}=\left(\frac{1}{\delta t\left|\nabla \theta^{n}\right|^{2}+1} \nabla \theta^{n}\right) \cdot \nabla \theta^{n}-\frac{1}{\delta t} \\
& =\frac{\left|\nabla \theta^{n}\right|^{2}}{\delta t\left|\nabla \theta^{n}\right|^{2}+1}-\frac{1}{\delta t}=\frac{\left|\nabla \theta^{n}\right|^{2}+\frac{1}{\delta t}-\frac{1}{\delta t}}{\delta t\left|\nabla \theta^{n}\right|^{2}+1}-\frac{1}{\delta t} \\
& =\frac{1}{\delta t}-\frac{1}{\delta t\left(\delta t\left|\nabla \theta^{n}\right|^{2}+1\right)}-\frac{1}{\delta t}=-\frac{1}{\delta t\left(\delta t\left|\nabla \theta^{n}\right|^{2}+1\right)} .
\end{aligned}
$$

Obviously, $a^{n}<0$. And we have

$$
\begin{aligned}
b^{n} & =a^{n} \theta^{n}+\left[\left(A^{n}\right)^{-1} \mathbf{u}^{n}\right] \cdot \nabla \theta^{n} \\
& =a^{n} \theta^{n}+\left\{\frac{1}{\delta t\left|\nabla \theta^{n}\right|^{2}+1}\left[I+\delta t\left(\theta_{y}^{n},-\theta_{x}^{n}\right)^{T}\left(\theta_{y}^{n},-\theta_{x}^{n}\right)\right] \mathbf{u}^{n}\right\} \cdot \nabla \theta^{n} \\
& =a^{n} \theta^{n}+\frac{1}{\delta t\left|\nabla \theta^{n}\right|^{2}+1} \mathbf{u}^{n} \cdot \nabla \theta^{n} .
\end{aligned}
$$

The weak form of (4.2) can be obtained by taking the inner product of both sides with a trial function and using the integration by parts:

Find $\theta^{n+1} \in H^{1}(\Omega)$, such that for any $\phi \in H^{1}(\Omega)$,

$$
-\left(\nabla \theta^{n+1}, \nabla \phi\right)+\left(a^{n} \theta^{n+1}, \phi\right)=\left(b^{n}, \phi\right) .
$$

For the equation (3.3), the corresponding weak formulation reads as follows. Find $\tilde{\mathbf{u}}^{n+1} \in$ $\left(H_{0}^{1}(\Omega)\right)^{2}$, such that for any $\mathbf{v} \in\left(H_{0}^{1}(\Omega)\right)^{2}$,

$$
\left(\frac{1}{\delta t} \tilde{\mathbf{u}}^{n+1}+\left(\mathbf{u}^{n} \cdot \nabla\right) \tilde{\mathbf{u}}^{n+1}, \mathbf{v}\right)+\left(\nabla \tilde{\mathbf{u}}^{n+1}, \nabla \mathbf{v}\right)=\left(\frac{1}{\delta t} \mathbf{u}_{*}^{n}, \mathbf{v}\right)-\left(\nabla \tilde{P}^{n}, \mathbf{v}\right) .
$$

For the pressure equation (3.5), the corresponding weak formulation reads as follows. Find $\tilde{P}^{n+1} \in H_{c}^{1}(\Omega) \triangleq\left\{P: P \in H^{1}(\Omega), \int_{\Omega} P d x=0\right\}$, such that for any $q \in H_{c}^{1}(\Omega)$,

$$
\left(\nabla\left(\tilde{P}^{n+1}-\tilde{P}^{n}\right), \nabla q\right)=-\frac{1}{\delta t}\left(\nabla \cdot \tilde{\mathbf{u}}^{n+1}, q\right)
$$

Remark 4.1. It is easy to prove that the above scheme avoids an inf-sup condition for the velocity and pressure [4].

\subsection{Finite element approximation}

Let $S_{h} \subset H^{1}(\Omega)$ and $M_{h} \subset L_{0}^{2}(\Omega)$ be the finite-dimensional subspaces constructed by the piecewise linear functions, where $L_{0}^{2}(\Omega) \triangleq\left\{q \in L^{2}(\Omega), \int_{\Omega} q d x=0\right\}$. Let $S_{h}^{0} \subset H_{0}^{1}(\Omega)$ and $V_{\mathbf{u}_{h}}=\left(S_{h}^{0}\right)^{2}$.

Thus, we rewrite the equations in finite element forms. 


\section{Step 1.}

Find $\theta_{h}^{n+1} \in S_{h}$, such that for any $\phi \in S_{h}$,

$$
\begin{aligned}
& -\left(\nabla \theta_{h}^{n+1}, \nabla \phi\right)+\left(a_{h}^{n} \theta_{h}^{n+1}, \phi\right)=\left(b_{h}^{n}, \phi\right), \\
& \left.\nabla \theta_{h}^{n+1} \cdot \mathbf{n}\right|_{\partial \Omega}=0,
\end{aligned}
$$

where

$$
\begin{aligned}
& a_{h}^{n}=\left[\left(A_{h}^{n}\right)^{-1} \nabla \theta_{h}^{n}\right] \cdot \nabla \theta_{h}^{n}-\frac{1}{\delta t}, \\
& b_{h}^{n}=\theta_{h}^{n}\left[\left(A_{h}^{n}\right)^{-1} \nabla \theta_{h}^{n}\right] \cdot \nabla \theta_{h}^{n}+\left[\left(A_{h}^{n}\right)^{-1} \mathbf{u}_{h}^{n}\right] \cdot \nabla \theta_{h}^{n}-\frac{1}{\delta t} \theta_{h}^{n}
\end{aligned}
$$

with $A_{h}^{n}=I+\delta t \nabla \theta_{h}^{n}\left(\nabla \theta_{h}^{n}\right)^{T}$.

Step 2.

Find $\tilde{\mathbf{u}}_{h}^{n+1} \in V_{\mathbf{u}_{h}}$, such that for any $\mathbf{v} \in V_{\mathbf{u}_{h}}$,

$$
\begin{aligned}
& \left(\frac{1}{\delta t} \tilde{\mathbf{u}}_{h}^{n+1}+\left(\mathbf{u}_{h}^{n} \cdot \nabla\right) \tilde{\mathbf{u}}_{h}^{n+1}, \mathbf{v}\right)+\left(\nabla \tilde{\mathbf{u}}_{h}^{n+1}, \nabla \mathbf{v}\right)=\left(\frac{1}{\delta t} \mathbf{u}_{* h}^{n}, \mathbf{v}\right)-\left(\nabla \tilde{P}_{h}^{n}, \mathbf{v}\right), \\
& \left.\tilde{\mathbf{u}}_{h}^{n+1}\right|_{\partial \Omega}=0
\end{aligned}
$$

where $\mathbf{u}_{* h}^{n}=\left(A_{h}^{n}\right)^{-1}\left[\mathbf{u}_{h}^{n}-\left(\theta_{h}^{n+1}-\theta_{h}^{n}\right) \nabla \theta_{h}^{n}\right]$.

\section{Step 3.}

Find $\tilde{P}_{h}^{n+1} \in M_{h}$, such that for any $q \in M_{h}$,

$$
\begin{aligned}
& \left(\nabla\left(\tilde{P}_{h}^{n+1}-\tilde{P}_{h}^{n}\right), \nabla q\right)=-\frac{1}{\delta t}\left(\nabla \cdot \tilde{\mathbf{u}}_{h}^{n+1}, q\right), \\
& \left.\nabla \tilde{P}_{h}^{n+1} \cdot \mathbf{n}\right|_{\partial \Omega}=0 .
\end{aligned}
$$

Then, we can obtain,

$$
\begin{aligned}
& \mathbf{u}_{h}^{n+1}=\tilde{\mathbf{u}}_{h}^{n+1}-\delta t \nabla\left(\tilde{P}_{h}^{n+1}-\tilde{P}_{h}^{n}\right), \\
& P_{h}^{n+1}=\tilde{P}_{h}^{n+1}-\frac{1}{2}\left|\nabla \theta_{h}^{n+1}\right|^{2} .
\end{aligned}
$$

Since each step in the scheme consists of a linear elliptic equation, the scheme is uniquely solvable.

Remark 4.2. Actually, $\theta_{h}^{n}$ should take values within $[0,2 \pi)$ for any $n \geqslant 1$. We omit this constraint in this section for simplicity. In Section 5, we prove that under some conditions, $\theta_{h}^{n} \in[0,2 \pi)$ for any $n \geqslant 1$ if $\theta^{0} \in[0,2 \pi)$, which shows that the constraint is satisfied.

\section{Discrete Maximum Principle}

In this section, we show that under some conditions, the above scheme in finite element form satisfies that $\theta_{h}^{n} \in[0,2 \pi)$, if $\theta^{0} \in[0,2 \pi)$, for any $n \geq 1$. According to Remark 2.2, it ensures 
the equivalence between system (2.6)-(2.8) and system (1.1)-(1.4) when performing numerical experiments. It is easy to see that, by mathematical induction, we only need to prove that $\theta_{h}^{n+1} \in[0,2 \pi)$, if $\theta_{h}^{n} \in[0,2 \pi)$, for any $n \geq 0$.

Assume that we use $P_{1}-P_{1}$ element for the velocity and pressure, and linear element for the function $\theta(\mathbf{x}, t)$, since the scheme avoids an inf-sup condition for the velocity and pressure. As in Section 4, denote $\left\{\phi_{i}\right\}_{i=1}^{N_{b}}$ as the piecewise linear basis for $S_{h}$. Having computed for $\theta_{h}^{n}, \mathbf{u}_{h}^{n}$, $P_{h}^{n}$ and $\tilde{P}_{h}^{n}=P_{h}^{n}+\frac{1}{2}\left|\nabla \theta_{h}^{n}\right|^{2}$ for $n>0$, we need to find $\theta_{h}^{n+1} \in S_{h}$, such that for any $\phi_{i} \in S_{h}$,

$$
-\left(\nabla \theta_{h}^{n+1}, \nabla \phi_{i}\right)+\left(a_{h}^{n} \theta_{h}^{n+1}, \phi_{i}\right)=\left(b_{h}^{n}, \phi_{i}\right), 1 \leq i \leq N_{b},
$$

where $a_{h}^{n}, b_{h}^{n}$ and $A_{h}^{n}$ are the same as those in Section 4. Namely,

$$
\begin{aligned}
& A_{h}^{n}=I+\delta t \nabla \theta_{h}^{n}\left(\nabla \theta_{h}^{n}\right)^{T}, \\
& \left(A_{h}^{n}\right)^{-1}=\frac{1}{\delta t\left|\nabla \theta_{h}^{n}\right|^{2}+1}\left[I+\delta t\left(\theta_{h y}^{n},-\theta_{h x}^{n}\right)^{T}\left(\theta_{h y}^{n},-\theta_{h x}^{n}\right)\right], \\
& a_{h}^{n}=-\frac{1}{\delta t\left(\delta t\left|\nabla \theta_{h}^{n}\right|^{2}+1\right)}, \\
& b_{h}^{n}=a_{h}^{n} \theta_{h}^{n}+\frac{1}{\delta t\left|\nabla \theta_{h}^{n}\right|^{2}+1} \mathbf{u}_{h}^{n} \cdot \nabla \theta_{h}^{n} .
\end{aligned}
$$

Denote $\theta_{h}^{n}=\sum_{j=1}^{N_{b}} x_{j}^{n} \phi_{j}$, then $\nabla \theta_{h}^{n}=\sum_{j=1}^{N_{b}} x_{j}^{n} \nabla \phi_{j}$, (5.1) can be rewritten as

$$
\begin{aligned}
& \sum_{j=1}^{N_{b}}\left[-\int_{\Omega} \nabla \phi_{j} \cdot \nabla \phi_{i} d \mathbf{x}+\int_{\Omega} a_{h}^{n} \phi_{j} \phi_{i} d \mathbf{x}\right] x_{j}^{n+1} \\
= & \sum_{j=1}^{N_{b}}\left[\int_{\Omega} a_{h}^{n} \phi_{j} \phi_{i} d \mathbf{x}+\int_{\Omega}\left(\frac{1}{\delta t\left|\nabla \theta_{h}^{n}\right|^{2}+1} \mathbf{u}_{h}^{n} \cdot \nabla \phi_{j}\right) \phi_{i} d \mathbf{x}\right] x_{j}^{n} .
\end{aligned}
$$

Therefore, (5.1) can be transformed into a linear equation

$$
\mathbf{M x}{ }^{n+1}=\mathbf{C x}^{n}
$$

where $\mathbf{M}=\left\{m_{i j}\right\}_{i, j=1}^{N_{b}}, \mathbf{C}=\left\{c_{i j}\right\}_{i, j=1}^{N_{b}}$ and $\mathbf{x}^{n}=\left(x_{1}^{n}, \cdots, x_{N_{b}}^{n}\right)^{T}$. Here,

$$
\begin{aligned}
& m_{i j}=-\int_{\Omega} \nabla \phi_{j} \cdot \nabla \phi_{i} d \mathbf{x}+\int_{\Omega} a_{h}^{n} \phi_{j} \phi_{i} d \mathbf{x}, \\
& c_{i j}=\int_{\Omega} a_{h}^{n} \phi_{j} \phi_{i} d \mathbf{x}+\int_{\Omega}\left(\frac{1}{\delta t\left|\nabla \theta_{h}^{n}\right|^{2}+1} \mathbf{u}_{h}^{n} \cdot \nabla \phi_{j}\right) \phi_{i} d \mathbf{x} .
\end{aligned}
$$

We first describe a lemma of our main result in this section, which is inspired by Lemma 3.1.1 from [33].

Lemma 5.1. Let $\mathbf{A}=\left\{a_{i j}\right\}_{i, j=1}^{n}, \mathbf{C}=\left\{c_{i j}\right\}_{i, j=1}^{n}$ be $n \times n$ matrices satisfying the conditions

(1) $\sum_{j=1}^{n} a_{i j}=\sum_{j=1}^{n} c_{i j}<0$ for $i=1, \cdots, n$,

(2) $c_{i j} \leq 0$ for $i, j=1, \cdots, n$,

(3) $a_{i j} \geq 0$ for $i=1, \cdots, n, j \neq i$, 
and assume that a $n$-dimensional vector $\mathbf{w}^{\prime}$ satisfies the linear equation

$$
\mathrm{Aw}^{\prime}=\mathbf{C w}
$$

where $\mathbf{w}$ is a n-dimensional vector. Then each component $w_{i}^{\prime}(i=1, \cdots, n)$ of $\mathbf{w}^{\prime}$ is estimated by

$$
w_{i}^{\prime} \geq \min _{j=1, \cdots, n} w_{j}, \quad w_{i}^{\prime} \leq \max _{j=1, \cdots, n} w_{j},
$$

where $w_{j}$ denotes the $j$-th component of $\mathbf{w}$.

Proof. Assume that $\min _{j=1, \cdots, n} w_{j}^{\prime}=w_{i}^{\prime}$. The $i$-th equation of (5.4) is written as

$$
a_{i i} w_{i}^{\prime}=\sum_{j \neq i}\left(-a_{i j}\right) w_{j}^{\prime}+\sum_{j=1}^{n} c_{i j} w_{j}
$$

Since the coefficients of $w_{j}^{\prime}$ and $w_{j}$ in the terms on the right-hand side of this equality are non-positive from the conditions (2) and (3), it holds that

$$
a_{i i} w_{i}^{\prime} \leq\left(-\sum_{j \neq i} a_{i j}\right) w_{i}^{\prime}+\left(\sum_{j=1}^{n} c_{i j}\right) \min _{j=1, \cdots, n} w_{j} .
$$

Namely,

$$
\left(\sum_{j=1}^{n} a_{i j}\right) w_{i}^{\prime} \leq\left(\sum_{j=1}^{n} c_{i j}\right) \min _{j=1, \cdots, n} w_{j}
$$

Since $\sum_{j=1}^{n} a_{i j}=\sum_{j=1}^{n} c_{i j}<0$, then we have $w_{i}^{\prime} \geq \min _{j=1, \cdots, n} w_{j}$, i.e., $\min _{j=1, \cdots, n} w_{j}^{\prime} \geq$ $\min _{j=1, \cdots, n} w_{j}$. Thus $w_{i}^{\prime} \geq \min _{j=1, \cdots, n} w_{j}$ for each $i=1, \cdots, n$. The latter half can be proved in the same manner.

Here, we consider the bounded domain $\Omega \subseteq \mathbb{R}^{2}$. Let $\mathcal{T}_{h}=\{e\}$ be a triangulation of $\Omega$ consisting of $N_{e}$ elements. The piecewise linear finite element space $S_{h}$ is defined as

$$
S_{h}=\left\{w_{h} \in C(\bar{\Omega}) ; w_{h} \text { is linear in each } e \in \mathcal{T}_{h}\right\} .
$$

We denote $P_{i}\left(i=1, \cdots, N_{b}\right)$ as the mesh nodes, and denote

$\Omega_{i}=\left\{e \in \mathcal{T}_{h} ; P_{i}\right.$ is one of the vertexes of element $\left.e\right\}$,

$N_{i}=$ number of elements in $\Omega_{i}, \quad N=\max _{i} N_{i}$,

$h_{i}^{e}=$ length of the edge opposite to the vertex $P_{i}$ in element $e, \quad h_{e}=\max _{P_{i} \in e} h_{i}^{e}, \quad h=\max _{e \in \mathcal{T}_{h}} h_{e}$,

$S_{e}=$ area of element $e, \quad S_{\max }=\max _{e \in \mathcal{T}_{h}} S_{e}, \quad S_{\min }=\min _{e \in \mathcal{T}_{h}} S_{e}$.

Denote the matrix $\mathbf{M}=\left\{m_{i j}\right\}_{i, j=1}^{N_{b}}$ as $\mathbf{M}=\mathbf{M}^{(1)}+\mathbf{M}^{(2)}$ with $\mathbf{M}^{(1)}=\left\{m_{i j}^{(1)}\right\}_{i, j=1}^{N_{b}}$ and $\mathbf{M}^{(2)}=\left\{m_{i j}^{(2)}\right\}_{i, j=1}^{N_{b}}$, where

$$
m_{i j}^{(1)}=-\int_{\Omega} \nabla \phi_{j} \cdot \nabla \phi_{i} d \mathbf{x}, \quad m_{i j}^{(2)}=\int_{\Omega} a_{h}^{n} \phi_{j} \phi_{i} d \mathbf{x} .
$$


The lumped mass method is employed here by replacing the mass matrix $\mathbf{M}^{(2)}$ by the diagonal matrix $\overline{\mathbf{M}}^{(2)}$, where $\bar{m}_{j j}^{(2)}=\sum_{k=1}^{N_{b}} m_{j k}^{(2)}$.

Thus we get to study the linear equation

$$
\overline{\mathbf{M}} \mathbf{x}^{n+1}=\mathbf{C} \mathbf{x}^{n},
$$

where $\overline{\mathbf{M}}=\mathbf{M}^{(1)}+\overline{\mathbf{M}}^{(2)}=\left\{\bar{m}_{i j}\right\}_{i, j=1}^{N_{b}}$, i.e.,

$$
\begin{aligned}
& \bar{m}_{i j}=m_{i j}^{(1)}=-\int_{\Omega} \nabla \phi_{j} \cdot \nabla \phi_{i} d \mathbf{x}, \quad \text { for } i \neq j, \\
& \bar{m}_{i i}=-\int_{\Omega}\left|\nabla \phi_{i}\right|^{2} d \mathbf{x}+\sum_{j=1}^{N_{b}} \int_{\Omega} a_{h}^{n} \phi_{j} \phi_{i} d \mathbf{x} .
\end{aligned}
$$

For the linear equation (5.6), since

$$
\sum_{j=1}^{N_{b}}\left(\nabla \phi_{j}, \nabla \phi_{i}\right)=\left(\nabla 1_{h}, \nabla \phi_{i}\right)=0
$$

where $1_{h}$ is the constant function whose value is equal to unity, it holds that

$$
\begin{aligned}
& \sum_{j=1}^{N_{b}} \bar{m}_{i j}=\sum_{j=1}^{N_{b}}\left(-\int_{\Omega} \nabla \phi_{j} \cdot \nabla \phi_{i} d \mathbf{x}+\int_{\Omega} a_{h}^{n} \phi_{j} \phi_{i} d \mathbf{x}\right)=\sum_{j=1}^{N_{b}} \int_{\Omega} a_{h}^{n} \phi_{j} \phi_{i} d \mathbf{x}, \\
& \sum_{j=1}^{N_{b}} c_{i j}=\sum_{j=1}^{N_{b}}\left(\int_{\Omega} a_{h}^{n} \phi_{j} \phi_{i} d \mathbf{x}+\int_{\Omega}\left(\frac{1}{\delta t\left|\nabla \theta_{h}^{n}\right|^{2}+1} \mathbf{u}_{h}^{n} \cdot \nabla \phi_{j}\right) \phi_{i} d \mathbf{x}\right)=\sum_{j=1}^{N_{b}} \int_{\Omega} a_{h}^{n} \phi_{j} \phi_{i} d \mathbf{x} .
\end{aligned}
$$

And $a_{h}^{n}=-\frac{1}{\delta t\left(\delta t\left|\nabla \theta_{h}^{n}\right|^{2}+1\right)}<0$ implies that

$$
\sum_{j=1}^{N_{b}} \bar{m}_{i j}=\sum_{j=1}^{N_{b}} c_{i j}<0, \quad \text { for } i=1, \cdots, N_{b}
$$

For $i \neq j$,

$$
\bar{m}_{i j}=m_{i j}^{(1)}=-\int_{\Omega} \nabla \phi_{j} \cdot \nabla \phi_{i} d \mathbf{x} .
$$

It has been proved that [32] if the triangulation $\mathcal{T}_{h}$ is of Delaunay type, $\left(\nabla \phi_{j}, \nabla \phi_{i}\right) \leq 0$, for $i \neq j$, i.e.,

$$
\bar{m}_{i j} \geq 0, \quad \text { for } \quad i \neq j \text {. }
$$

For the matrix $\mathbf{C}$, since we use the $P_{1}$ element for the velocity, denote $\mathbf{u}_{h}^{n}=\sum_{k=1}^{N_{b}} \mathbf{u}_{h k}^{n} \phi_{k}$, where $\mathbf{u}_{h k}^{n}=\left(u_{1 h k}^{n}, u_{2 h k}^{n}\right)^{T}$. Then

$$
\begin{aligned}
c_{i j} & =\int_{\Omega} a_{h}^{n} \phi_{j} \phi_{i} d \mathbf{x}+\int_{\Omega} \frac{1}{\delta t\left|\nabla \theta_{h}^{n}\right|^{2}+1}\left(\sum_{k=1}^{N_{b}} u_{1 h k}^{n} \phi_{k} \phi_{j x}+\sum_{k=1}^{N_{b}} u_{2 h k}^{n} \phi_{k} \phi_{j y}\right) \phi_{i} d \mathbf{x} \\
& \triangleq c_{i j}^{(1)}+c_{i j}^{(2)} .
\end{aligned}
$$

For $c_{i j}^{(2)}$ with $i \neq j$, we have

$$
c_{i j}^{(2)}=\sum_{e \in \Omega_{i} \cap \Omega_{j}} \int_{e} \frac{1}{\delta t\left|\nabla \theta_{h}^{n}\right|^{2}+1}\left[\sum_{k=1}^{N_{b}}\left(u_{1 h k}^{n} \phi_{k} \phi_{j x}+u_{2 h k}^{n} \phi_{k} \phi_{j y}\right)\right] \phi_{i} d \mathbf{x} .
$$


For $e \in \Omega_{i} \cap \Omega_{j}$, assume that the vertexes of $e$ are $P_{i}, P_{j}$ and $P_{m}, i \neq j$, due to $\frac{1}{\delta t\left|\nabla \theta_{h}^{n}\right|^{2}+1} \leq 1$, it holds that

$$
\begin{aligned}
c_{i j}^{(2)} & =\sum_{e \in \Omega_{i} \cap \Omega_{j}} \int_{e} \frac{1}{\delta t\left|\nabla \theta_{h}^{n}\right|^{2}+1}\left[\sum_{l=i, j, m}\left(u_{1 h l}^{n} \phi_{l} \phi_{j x}+u_{2 h l}^{n} \phi_{l} \phi_{j y}\right)\right] \phi_{i} d \mathbf{x} \\
& \leq \sum_{e \in \Omega_{i} \cap \Omega_{j}} \int_{e}\left|\sum_{l=i, j, m}\left(u_{1 h l}^{n} \phi_{l} \phi_{j x}+u_{2 h l}^{n} \phi_{l} \phi_{j y}\right) \phi_{i}\right| d \mathbf{x} \\
& \leq \sum_{e \in \Omega_{i} \cap \Omega_{j}} \int_{e} \sum_{l=i, j, m}\left|u_{1 h l}^{n}\right|\left|\phi_{j x}\right| \phi_{l} \phi_{i}+\left|u_{2 h l}^{n}\right|\left|\phi_{j y}\right| \phi_{l} \phi_{i} d \mathbf{x} .
\end{aligned}
$$

By simple calculation, we have,

$$
\int_{e} \phi_{l} \phi_{i} d \mathbf{x}= \begin{cases}\frac{S_{e}}{12}, & \text { for } l=j \text { or } m \\ \frac{S_{e}}{6}, & \text { for } l=i .\end{cases}
$$

And in the element $e$,

$$
\left|\nabla \phi_{j}\right|=\frac{1}{2 S_{e}} h_{j} .
$$

Hence, $\left|\phi_{j x}\right| \leq\left|\nabla \phi_{j}\right|=\frac{1}{2 S_{e}} h_{j},\left|\phi_{j y}\right| \leq\left|\nabla \phi_{j}\right|=\frac{1}{2 S_{e}} h_{j}$. Since there exists two elements in $\Omega_{i} \cap \Omega_{j}$, it holds that,

$$
\begin{aligned}
c_{i j}^{(2)} & \leq \sum_{e \in \Omega_{i} \cap \Omega_{j}}\left[\sum_{l=i, j, m}\left(\left|u_{1 h l}^{n}\right|+\left|u_{2 h l}^{n}\right|\right) \frac{S_{e}}{6} \times \frac{1}{2 S_{e}} h_{j}\right] \\
& \leq \sum_{e \in \Omega_{i} \cap \Omega_{j}} \frac{1}{2} h_{e} \max _{k=1, \ldots, N_{b}}\left\{\left|u_{1 h k}^{n}\right|,\left|u_{2 h k}^{n}\right|\right\} \\
& \leq h \max _{k=1, \ldots, N_{b}}\left\{\left|u_{1 h k}^{n}\right|,\left|u_{2 h k}^{n}\right|\right\} .
\end{aligned}
$$

For $c_{i i}^{(2)}$, assume that $e \in \Omega_{i}$ and the vertexes of $e$ are $P_{i}, P_{l}$ and $P_{m}$. Then we have

$$
\begin{aligned}
c_{i i}^{(2)} & =\sum_{e \in \Omega_{i}} \int_{e} \frac{1}{\delta t\left|\nabla \theta_{h}^{n}\right|^{2}+1}\left[\sum_{q=i, l, m}\left(u_{1 h q}^{n} \phi_{q} \phi_{i x}+u_{2 h q}^{n} \phi_{q} \phi_{i y}\right)\right] \phi_{i} d \mathbf{x} \\
& \leq \sum_{e \in \Omega_{i}} \int_{e}\left|\sum_{q=i, l, m}\left(u_{1 h q}^{n} \phi_{q} \phi_{i x}+u_{2 h q}^{n} \phi_{q} \phi_{i y}\right) \phi_{i}\right| d \mathbf{x} \\
& \leq \sum_{e \in \Omega_{i}} \sum_{q=i, l, m}\left(\left|u_{1 h q}^{n}\right|+\left|u_{2 h q}^{n}\right|\right) \times \frac{S_{e}}{6} \times \frac{1}{2 S_{e}} h_{i} \\
& \leq \sum_{e \in \Omega_{i}} \frac{1}{2} h_{e} \max _{k=1, \ldots, N_{b}}\left\{\left|u_{1 h k}^{n}\right|,\left|u_{2 h k}^{n}\right|\right\} \\
& \leq \frac{N}{2} h \max _{k=1, \ldots, N_{b}}\left\{\left|u_{1 h k}^{n}\right|,\left|u_{2 h k}^{n}\right|\right\} .
\end{aligned}
$$

For $c_{i j}^{(1)}$ with $i \neq j$,

$$
\begin{aligned}
c_{i j}^{(1)} & =\int_{\Omega}-\frac{1}{\delta t\left(\delta t\left|\nabla \theta_{h}^{n}\right|^{2}+1\right)} \phi_{j} \phi_{i} d \mathbf{x} \\
& =\sum_{e \in \Omega_{i} \cap \Omega_{j}} \int_{e}-\frac{1}{\delta t\left(\delta t\left|\nabla \theta_{h}^{n}\right|^{2}+1\right)} \phi_{j} \phi_{i} d \mathbf{x} .
\end{aligned}
$$


For the element $e \in \Omega_{i} \cap \Omega_{j}$, assume that the vertexes of $e$ are $P_{i}, P_{j}$ and $P_{m}$ as before. Due to $\theta_{h}^{n}=\sum_{k=1}^{N_{b}} x_{k}^{n} \phi_{k}, \nabla \theta_{h}^{n}=\sum_{k=1}^{N_{b}} x_{k}^{n} \nabla \phi_{k}$, Eq. (5.10) and (5.11), it holds that

$$
\begin{aligned}
\left.\left|\nabla \theta_{h}^{n}\right|^{2}\right|_{e} & =\left.\left|x_{i}^{n} \nabla \phi_{i}+x_{j}^{n} \nabla \phi_{j}+x_{m}^{n} \nabla \phi_{m}\right|^{2}\right|_{e} \\
& \leq\left. 2\left(\left|x_{i}^{n} \nabla \phi_{i}\right|^{2}+\left|x_{j}^{n} \nabla \phi_{j}\right|^{2}+\left|x_{m}^{n} \nabla \phi_{m}\right|^{2}\right)\right|_{e} \\
& =\left.2\left(\left|x_{i}^{n}\right|^{2}\left(\frac{1}{2 S_{e}} h_{i}\right)^{2}+\left|x_{j}^{n}\right|^{2}\left(\frac{1}{2 S_{e}} h_{j}\right)^{2}+\left|x_{m}^{n}\right|^{2}\left(\frac{1}{2 S_{e}} h_{m}\right)^{2}\right)\right|_{e} \\
& \leq 2 X^{n}\left(\frac{1}{2 S_{e}}\right)^{2}\left(h_{i}^{2}+h_{j}^{2}+h_{k}^{2}\right) \\
& \leq \frac{3 h_{e}^{2} X^{n}}{2 S_{e}^{2}} \leq \frac{3 h^{2} X^{n}}{2 S_{\min }^{2}}
\end{aligned}
$$

where $X^{n} \triangleq \max _{k=1, \ldots, N_{b}}\left|x_{k}^{n}\right|^{2}$. And then

$$
\begin{aligned}
c_{i j}^{(1)} & \leq \sum_{e \in \Omega_{i} \cap \Omega_{j}} \int_{e}-\frac{1}{\delta t\left(\delta t \frac{3 h^{2} X^{n}}{2 S_{\min }^{2}}+1\right)} \phi_{j} \phi_{i} d \mathbf{x} \\
& =\sum_{e \in \Omega_{i} \cap \Omega_{j}}-\frac{2 S_{\min }^{2}}{\delta t\left(3 h^{2} \delta t X^{n}+2 S_{\min }^{2}\right)} \cdot \frac{1}{12} S_{e} \\
& \leq \sum_{e \in \Omega_{i} \cap \Omega_{j}}-\frac{2 S_{\min }^{2}}{\delta t\left(3 h^{2} \delta t X^{n}+2 S_{\min }^{2}\right)} \cdot \frac{1}{12} S_{\min } .
\end{aligned}
$$

Since there exists two elements in $\Omega_{i} \cap \Omega_{j}$,

$$
c_{i j}^{(1)} \leq-\frac{2 S_{\min }^{2}}{\delta t\left(3 h^{2} \delta t X^{n}+2 S_{\min }^{2}\right)} \cdot \frac{1}{6} S_{\min }=-\frac{S_{\min }^{3}}{3 \delta t\left(3 h^{2} \delta t X^{n}+2 S_{\min }^{2}\right)} .
$$

Similarly, for $i=j$, since $N_{i} \geq 1$,

$$
\begin{aligned}
c_{i i}^{(1)} & \leq \sum_{e \in \Omega_{i}} \int_{e}-\frac{1}{\delta t\left(\delta t \frac{3 h^{2} X^{n}}{2 S_{\min }^{2}}+1\right)} \phi_{i}^{2} d \mathbf{x}=\sum_{e \in \Omega_{i}}-\frac{2 S_{\min }^{2}}{\delta t\left(3 h^{2} \delta t X^{n}+2 S_{\min }^{2}\right)} \cdot \frac{1}{6} S_{e} \\
& \leq \sum_{e \in \Omega_{i}}-\frac{S_{\min }^{3}}{3 \delta t\left(3 h^{2} \delta t X^{n}+2 S_{\min }^{2}\right)}=-\frac{N_{i} S_{\min }^{3}}{3 \delta t\left(3 h^{2} \delta t X^{n}+2 S_{\min }^{2}\right)} \\
& \leq-\frac{S_{\min }^{3}}{3 \delta t\left(3 h^{2} \delta t X^{n}+2 S_{\min }^{2}\right)} .
\end{aligned}
$$

Combine Eq. (5.12)-(5.15), and due to the fact that $N \geq 2$, it holds that, for $i, j=1, \ldots, N_{b}$,

$$
\begin{aligned}
c_{i j} & =c_{i j}^{(1)}+c_{i j}^{(2)} \\
& \leq-\frac{S_{\min }^{3}}{3 \delta t\left(3 h^{2} \delta t X^{n}+2 S_{\min }^{2}\right)}+\frac{N}{2} h \max _{k=1, \ldots, N_{b}}\left\{\left|u_{1 h k}^{n}\right|,\left|u_{2 h k}^{n}\right|\right\} .
\end{aligned}
$$

Assume that $\theta_{h}^{n} \in[0,2 \pi)$, i.e., $x_{k}^{n}=\theta_{h}^{n}\left(P_{k}\right) \in[0,2 \pi)$, for $k=1, \cdots, N_{b}$. Then it holds that $X^{n}=\max _{k=1, \ldots, N_{b}}\left|x_{k}^{n}\right|^{2}=(2 \pi)^{2}=4 \pi^{2}$, and

$$
\begin{aligned}
c_{i j} & \leq-\frac{S_{\min }^{3}}{3 \delta t\left(3 h^{2} \delta t \cdot 4 \pi^{2}+2 S_{\min }^{2}\right)}+\frac{N}{2} h \max _{k=1, \ldots, N_{b}}\left\{\left|u_{1 h k}^{n}\right|,\left|u_{2 h k}^{n}\right|\right\} \\
& =-\frac{S_{\min }^{3}}{6 \delta t\left(6 \pi^{2} h^{2} \delta t+S_{\min }^{2}\right)}+\frac{N}{2} h \max _{k=1, \ldots, N_{b}}\left\{\left|u_{1 h k}^{n}\right|,\left|u_{2 h k}^{n}\right|\right\} .
\end{aligned}
$$


Therefore, $c_{i j} \leq 0, i, j=1, \cdots, N_{b}$, under the condition that

$$
\max _{k=1, \ldots, N_{b}}\left\{\left|u_{1 h k}^{n}\right|,\left|u_{2 h k}^{n}\right|\right\} \leq \frac{S_{\min }^{3}}{3 N h \delta t\left(6 \pi^{2} h^{2} \delta t+S_{\min }^{2}\right)} .
$$

Our main result is stated in the following theorem.

Theorem 5.1. For the linear equation (5.6), if the triangulation $\mathcal{T}_{h}$ is of Delaunay type, under the condition (5.17), $\overline{\mathbf{M}}=\left\{\bar{m}_{i j}\right\}_{i, j=1}^{N_{b}}$ and $\mathbf{C}=\left\{c_{i j}\right\}_{i, j=1}^{N_{b}}$ satisfy the conditions

(1) $\sum_{j=1}^{N_{b}} \bar{m}_{i j}=\sum_{j=1}^{N_{b}} c_{i j}<0$ for $i=1, \cdots, N_{b}$,

(2) $c_{i j} \leq 0$ for $i, j=1, \cdots, N_{b}$,

(3) $\bar{m}_{i j} \geq 0$ for $i=1, \cdots, N_{b}, j \neq i$.

If $\mathbf{x}^{n}=\left(x_{1}^{n}, \cdots, x_{N_{b}}^{n}\right)^{T}$ satisfies $x_{i}^{n} \in[0,2 \pi)$, for $i=1, \cdots, N_{b}$, then $x_{i}^{n+1} \in[0,2 \pi)$, where $x_{i}^{n+1}$ denotes the $i$-th component of $\mathbf{x}^{n+1}$.

Proof. From (5.7) and (5.8), conditions (1) and (3) follow at once. From (5.16), condition (2) is satisfied if (5.17) holds true. The rest of the proof is similar to that of Lemma 5.1.

Remark 5.1. In Theorem 5.1, we prove that $\theta_{h}^{n+1} \in[0,2 \pi)$, if $\theta_{h}^{n} \in[0,2 \pi)$, for any $n \geq 0$, under conditions that the triangulation $\mathcal{T}_{h}$ is of Delaunay type and Eq. (5.17) holds true.

Remark 5.2. Since $S_{\min }=\mathcal{O}\left(h^{2}\right)$, assume that $\delta t=\mathcal{O}\left(h^{\alpha}\right)$, Eq. (5.17) can be rewritten as

$$
\max _{k=1, \ldots, N_{b}}\left\{\left|u_{1 h k}^{n}\right|,\left|u_{2 h k}^{n}\right|\right\} \leq \mathcal{O}\left(\frac{1}{3 N\left(6 \pi^{2} h^{2 \alpha-3}+h^{\alpha-1}\right)}\right) .
$$

In order to make sure that the analysis is applicable for non-trivial cases when $h \rightarrow 0$, the condition that $\alpha \geq \frac{3}{2}$ should hold true.

Remark 5.3. Condition (5.17) requires that $\mathbf{u}_{h}^{n}$ should take values satisfying

$$
\max _{k=1, \ldots, N_{b}}\left\{\left|u_{1 h k}^{n}\right|,\left|u_{2 h k}^{n}\right|\right\} \leq \frac{S_{\min }^{3}}{3 N h \delta t\left(6 \pi^{2} h^{2} \delta t+S_{\min }^{2}\right)},
$$

to make sure that $\theta_{h}^{n} \in[0,2 \pi)$, for any $n \geq 0$. However, it is actually not necessary in specific numerical experiments since the estimate of $\theta_{h}^{n}$ and $\nabla \theta_{h}^{n}$ here is coarse. In this paper, we choose the suitable mesh size and time step when performing numerical experiments, so that the discrete maximum principle is satisfied, i.e., the function $\theta_{h}^{n} \in[0,2 \pi)$. Evidence for the discrete maximum principle of the numerical solutions is provided in Section 6.

\section{Numerical Experiments}

In this section, we will show numerical experiments to validate the scheme constructed above. We simulate the smooth solutions of (1.1)-(1.4) by solving (2.6)-(2.8) and the numerical results are in good qualitative agreement with those obtained in [3]. We use the piecewise linear element for the function $\theta(\mathbf{x}, t)$ and the $P_{1}-P_{1}$ element for the velocity and pressure, since the scheme avoids an inf-sup condition for the velocity and pressure. All our experiments are computed on 
the domain $\Omega=(0,1)^{2}$ and $\mathcal{T}_{h}$ is a uniform triangulation, where each element is an isosceles right triangle. According to the analysis in Section 5, we choose the suitable mesh size and time step, so that the discrete maximum principle is satisfied, i.e., the function $\theta_{h}^{n} \in[0,2 \pi)$ in all the numerical experiments. Evidence for the discrete maximum principle is also shown in this section.

We consider the system (2.6)-(2.8) with the initial conditions:

$$
\mathbf{u}_{0} \equiv \mathbf{0}, \quad \mathbf{d}_{0}=(\cos (a), \sin (a))^{T}, \quad a=2 \pi(\cos (x)-\sin (y)),
$$

i.e.,

$$
\theta_{0}= \begin{cases}2 \pi+a, & \text { if } \cos (x)-\sin (y)<0, \\ 0, & \text { if } \cos (x)-\sin (y)=1, \\ a, & \text { else. }\end{cases}
$$

We choose the mesh size $h=1 / 50$, and the time step size $\delta t=0.001$. The numerical results of d are shown in Fig. 6.1.

The maximum and minimum values of the numerical solutions $\theta_{h}^{n}$ are plotted as functions of $n$ to validate the discrete maximum principle. It is shown in Figs. 6.2 and 6.3 that $\theta_{h}^{n} \in[0,2 \pi)$ for $n=0, \ldots, 1000$ and when $n \geq 400$, the maximum and minimum values of $\theta_{h}^{n}$ almost remain the same, which indicates that the system almost reaches a steady state. Thus, it is reasonable to conclude that $\theta_{h}^{n} \in[0,2 \pi)$ for all $n \in \mathbb{N}$.

In order to measure the time error, we choose the reference solution as the numerical approximation computed on the discrete parameters $(h, \delta t)=(0.02,0.0005)$. In Figs. 6.6 and 6.7, we plot the $L^{2}$ errors of the velocity, pressure, and the function $\theta$ between the numerical solution and the reference solution at $t=1$ with different time step size $\delta t=0.005,0.01,0.02,0.04$ and
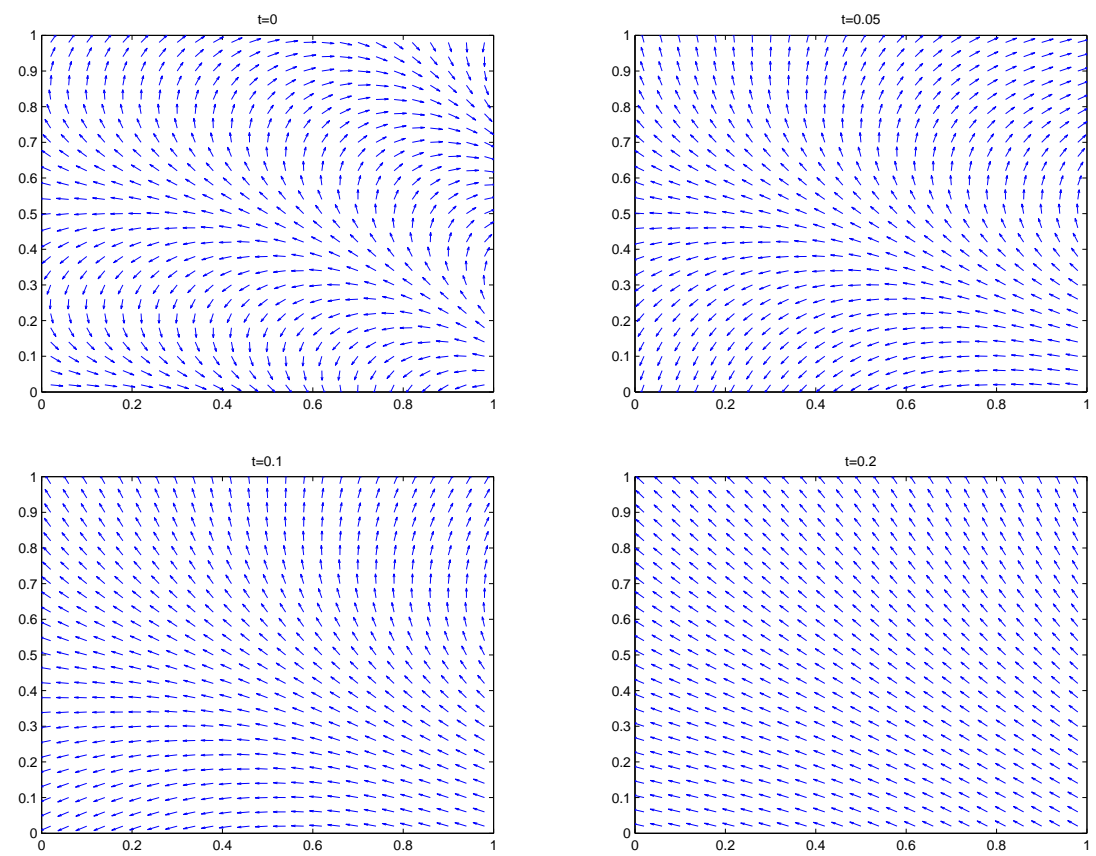

Fig. 6.1. Numerical results of $\mathbf{d}$. 

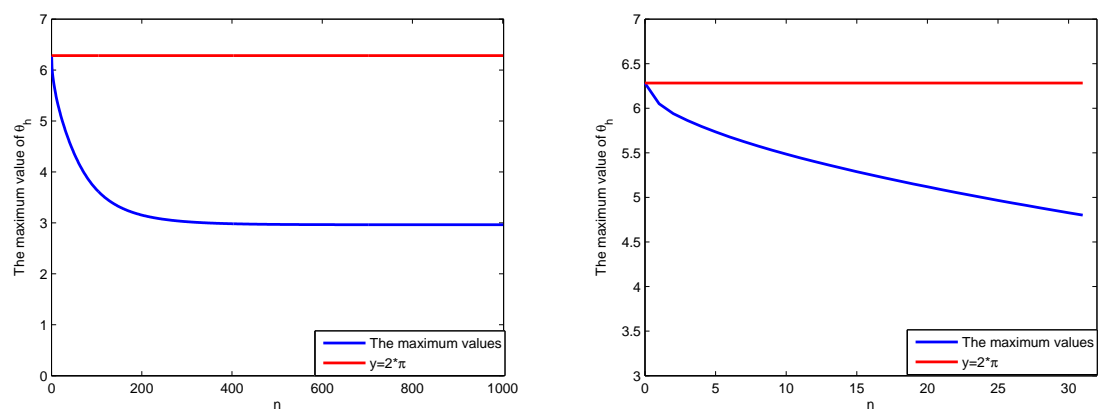

Fig. 6.2. The maximum values of $\theta_{h}^{n}$ for different $n$ (left) and the local magnification (right).

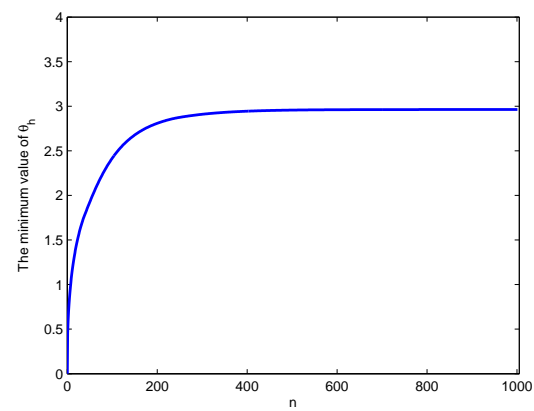

Fig. 6.3. The minimum values of $\theta_{h}^{n}$ for different $n$.
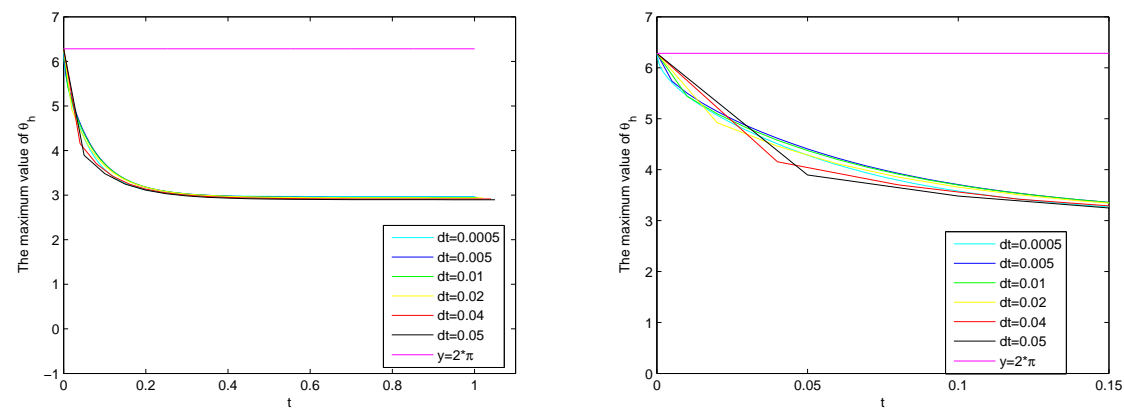

Fig. 6.4. The maximum values of $\theta_{h}^{n}$ for different $\delta t$ (left) and the local magnification (right).

0.05. The maximum and minimum values of the numerical solutions $\theta_{h}^{n}$ are plotted in Figs. 6.4 and 6.5 as an evidence to validate the discrete maximum principle for these different time steps. The numerical results show that scheme (3.1)-(3.7) is first-order accurate in time for all variables.

\section{Conclusions and Remarks}

In this paper, we rewrite the 2D simplified Ericksen-Leslie system (1.1)-(1.4) and propose a new system (2.6)-(2.8). We construct a time discretization numerical scheme for the new system. The scheme is the first constraint-preserving, decoupled and energy-stable scheme for 


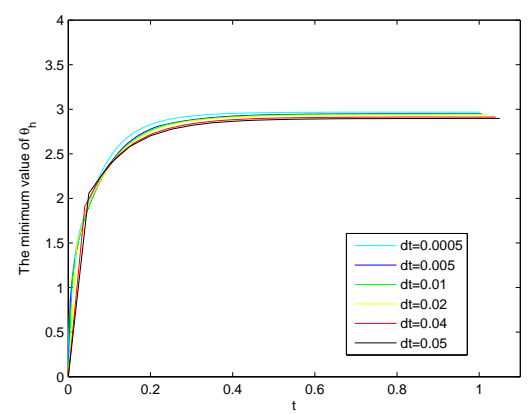

Fig. 6.5. The minimum values of $\theta_{h}^{n}$ for different $\delta t$.
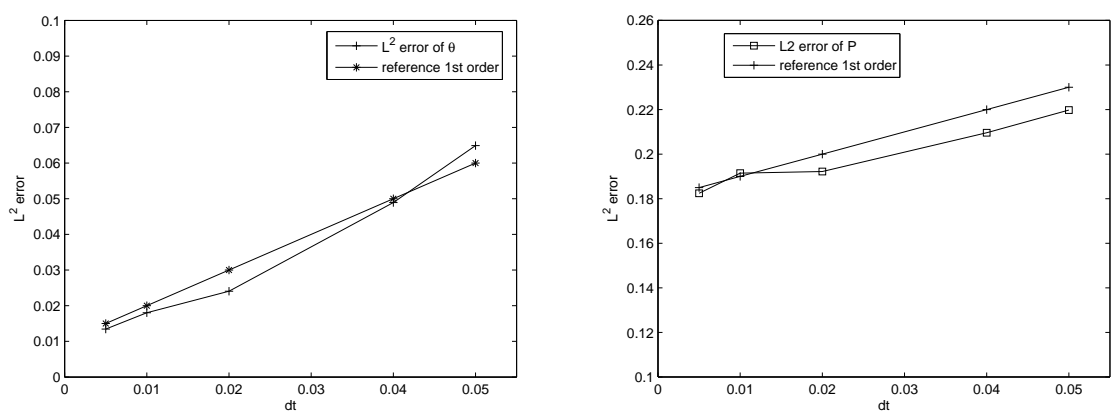

Fig. 6.6. $L^{2}$ errors of the function $\theta$ (left) and pressure(right).

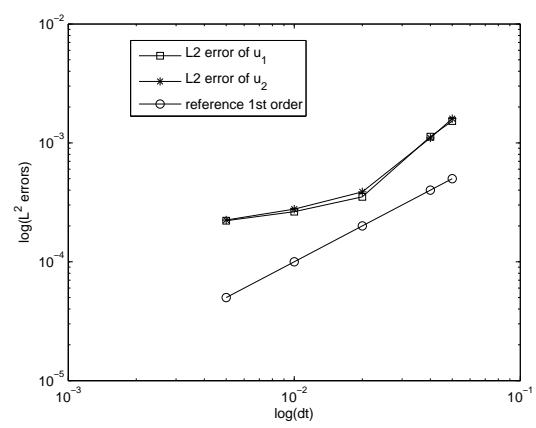

Fig. 6.7. $L^{2}$ errors of the velocity $\left(\mathbf{u}=\left(u_{1}, u_{2}\right)\right)$.

the $2 \mathrm{D}$ simplified Ericksen-Leslie system so far. The scheme is quite easy to implement. One only needs to solve linear and decoupled elliptic equations at each time step. It also satisfies the discrete maximum principle, which ensures the equivalence between system (2.6)-(2.8) and system (1.1)-(1.4) when performing numerical experiments. Numerical experiments are presented to validate the scheme and simulate the dynamic motion of liquid crystals.

Acknowledgement. H. Zhang is partially supported by NSFC grant No. 11471046, 11571045. R. Chen is partially supported by the Fundamental Research Funds for Central Universities No. 24820182018RC25-500418780. 


\section{References}

[1] S. Badia, F. Guillén-González and J.V. Gutiérrez-Santacreu, An overview on numerical analyses of nematic liquid crystal flows, Arch. Comput. Methods Eng., 18 (2011), 285-313.

[2] S. Bartels and A. Prohl, Constraint preserving implicit finite element discretization of harmonic map flow into spheres, Math. Comp., 76 (2007), 1847-1859.

[3] R. Becker, X.B. Feng and A. Prohl, Finite element approximations of the Ericksen-Leslie model for nematic liquid crystal flow, SIAM J. Numer. Anal., 46 (2008), 1704-1731.

[4] D. Boffi, F. Brezzi and M. Fortin, Mixed Finite Element Methods and Applications, Springer, Heidelberg, 2013.

[5] R.C. Cabrales, F. Guillén-González and J. V. Gutiérrez-Santacreu, A time-splitting finite-element stable approximation for the Ericksen-Leslie equations, SIAM J. Sci. Comput., 37 (2015), 261-282.

[6] K.C. Chang, W.Y. Ding and R.G. Ye, Finite-time blow-up of the heat flow of harmonic maps from surfaces, J. Differential Geom., 36 (1992), 507-515.

[7] R. Chen, G.H. Ji, X.F. Yang and H. Zhang, Decoupled energy stable schemes for phase-field vesicle membrane model, J. Comput. Phys., 302 (2015), 509-523.

[8] R. Chen, W.Z. Bao and H. Zhang, The kinematic effects of the defects in liquid crystal dynamics, Commun. Comput. Phys., 20 (2016), 234-249.

[9] Y. Chen and Y. Yu, Global m-equivariant solutions of nematic liquid crystal flows in dimension two, Arch. Ration. Mech. Anal., 226 (2017), 767-808.

[10] Y. Chen, S. Kim and Y. Yu, Twisted solutions to a simplified Ericksen-Leslie equation, Arch. Ration. Mech. Anal., 232 (2019), 303-336.

[11] P.A. Cruz, M.F. Tomé, I.W. Stewart and S. McKee, Numerical solution of the Ericksen-Leslie dynamic equations for two-dimensional nematic liquid crystal flows, J. Comput. Phys., 247 (2013), 109-136.

[12] J.L. Ericksen, Hydrostatic theory of liquid crystals, Arch. Rational Mech. Anal., 9 (1962), 371-378.

[13] A. Fuwa and T. Ishiwata, Finite difference scheme for the Ericksen-Leslie equation, Proceedings of Czech-Japanese Seminar in Applied Mathematics 2010, 26-35, COE Lect. Note, 36, Math-for-Ind, (MI) Lect. Note Ser., Kyushu Univ. Fac. Math., Fukuoka, 2012.

[14] V. Girault and F. Guillén-González, Mixed formulation, approximation and decoupling algorithm for a penalized nematic liquid crystals model, Math. Comp., 80 (2011), 781-819.

[15] H.J. Gong, J.R. Huang, L.M. Liu and X.G. Liu, Gobal strong solutions of the 2D simplified Ericksen-Leslie system, Nonlinearity, 28 (2015), 3677-3694.

[16] J.L. Guermond, P. Minev and J. Shen, An overview of projection methods for incompressible flows, Comput. Methods Appl. Mech. Engrg., 195 (2006), 6011-6045.

[17] M.C. Hong, Global existence of solutions of the simplified Ericksen-Leslie system in dimension two, Calc. Var. Partial Differential Equations, 40 (2011), 15-36.

[18] T. Huang, F.H. Lin, C. Liu and C.Y. Wang, Finite time singularity of the nematic liquid crystal flow in dimension three, Arch. Ration. Mech. Anal., 221 (2016), 1223-1254.

[19] X.D. Huang, A representation formula of incompressible liquid crystal flow and its applications, Nonlinearity, 30 (2017), 1911-1919.

[20] F.M. Leslie, Some constitutive equations for liquid crystals, Arch. Rational Mech. Anal., 28 (1968), 265-283.

[21] F.H. Lin, Nonlinear theory of defects in nematic liquid crystals; phase transition and flow phenomena, Comm. Pure Appl. Math., 42 (1989), 789-814.

[22] F.H. Lin and C. Liu, Nonparabolic dissipative systems modeling the flow of liquid crystals, Comm. Pure Appl. Math., 48 (1995), 501-537.

[23] F.H. Lin and C. Liu, Partial regularity of the dynamic system modeling the flow of liquid crystals, Discrete Contin. Dynam. Systems, 2 (1996), 1-22.

[24] F.H. Lin and C. Liu, Existence of solutions for the Ericksen-Leslie system, Arch. Ration. Mech. 
Anal., 154 (2000), 135-156.

[25] F.H. Lin, J.Y. Lin and C.Y. Wang, Liquid crystal flows in two dimensions, Arch. Ration. Mech. Anal., 197 (2010), 297-336.

[26] F.H. Lin and C.Y. Wang, On the uniqueness of heat flow of harmonic maps and hydrodynamic flow of nematic liquid crystals, Chin. Ann. Math. Ser. B, 31 (2010), 921-938.

[27] F.H. Lin and C.Y. Wang, Recent developments of analysis for hydrodynamic flow of nematic liquid crystals, Philos. Trans. R. Soc. Lond. Ser. A Math. Phys. Eng. Sci., 372, (2014), 18 pp.

[28] C. Liu and N.J. Walkington, Approximation of liquid crystal flows, SIAM J. Numer. Anal., 37 (2000), 725-741.

[29] C. Liu and N.J. Walkington, Mixed methods for the approximation of liquid crystal flows, Math. Model. Numer. Anal., 36 (2002), 205-222.

[30] M.H. Protter and H.F. Weinberger, Maximum Principles in Differential Equations, Prentice-Hall, Inc., Englewood Cliffs, N. J., 1967.

[31] R. Temam, Navier-Stokes Equations. Theory and Numerical Analysis, North-Holland Publishing Co., Amsterdam-New York-Oxford, 1977.

[32] V. Thomée, Galerkin Finite Element Methods for Parabolic Problems, 2nd ed, Springer Series in Computational Mathematics 25, 1997.

[33] I. Tsutomu, Maximum Principle in Finite Element Models for Convection-Diffusion Phenomena, Book Store Co., Ltd., Tokyo, 1983. 